\title{
EXISTENCE THEOREMS FOR DIFFERENTIAL INCLUSIONS WITH NONCONVEX RIGHT HAND SIDE
}

\author{
NIKOLAOS S. PAPAGEORGIOU \\ University of Illinoi:; \\ Department of Mathematics \\ 1409 West Green Street \\ Urbana, Illinois 61801
}

(Received November 21, 1985 and in revised form February 26, 1986)

\begin{abstract}
In this paper we prote some new existence theorems for differential inclusions with a nonconvex right hand side, which is Jower semicontinuous or continuous in the state variable, measurable in the time variable and takes valucs in a $f$ inite or infinite dimensional separable Banach space.
\end{abstract}

1980 Mathematics Subject Classification: 34605

KEY WORDS AND PIRASES: Orienter field, multifunction, measure of noncompactness, measurable rultifunction, lower scmicontinuous multifunction, Haustorff metric, Kamke function.

1. INTRODUCTION.

In the recent years there has been an increase in interest in the investigation of systems described by differential inclusions. In any orcinary differential equation the tangent at each point is prescribed by a single valued function. In a differential inclusion the tangent is prescribcd by a multifunction (set valued function) which is usually called an orientor field. "any problems of afplicd inathomatics lead us to the study of dymamical systcms having velocities not uniquely determined by the state of the system, but depending only luosciy upon it. In these cases the classical equation $\dot{x}(t)=f(t, x(t))$ describing the dynamics of the system is replaced by a relation of the form $\dot{x}(t) \in F(t, x(t))$ where $F(\cdot, \cdot)$ is a multifunction (the orientor field). Such a "set valued differential equation" is called "differential inclusion". The initial impetus to study differential inclusions came from control theory. Then the subject found adidional important applications in mathenatical economics [1], nonsmooth dynamics [2], optimization [3], differential equations with a discontinuous forcing term $\left[\frac{1}{2}\right]$ etc. 
The purpose of this paper is to prove existence theorems for differential inclusions governed by nonconvex valued, lower semicontinuous orientor fields which take values in a separable Banach space. Until now, most of the existence theory for differential inclusions was developed for upper semicontinuous, convex valued orientor fields with values in $\mathbb{R}^{n}$. However lower semicontinuous, nonconvex valued orientor fields appear of ten in control theory in connection with the barig-bang principle. So it is important to have existence theorems for differential inclusions governed by such orientor fields.

2. PRELIMINARIES.

Let $(\Omega, \Sigma)$ be a measurable space and let $X$ be a separable Banach space, with $X^{*}$ being its topological dual. We will use the following notation.

$$
P_{f}(X)=\{A \subseteq X: \text { nonempty, closed }\}
$$

For $A \in 2^{X} \backslash(\phi\}$, we set $|A|=\sup _{X \in A}\|x\|$ and by $d_{A}(\cdot)$ we denote the distance function from $A$ i.e. for all $x \in X, d_{A}(x)=\underset{a \in A}{\inf } \| x-a l l$

A multifunction $F: \Omega \rightarrow P_{f}(X)$ is said to be measurable if it satisfies any of the following equivalent conditions.

(i) $\omega \rightarrow d(x)$ is measurable for all $x \in X$ $F(\omega)$

(ii) there exists a sequence $\left\{f_{n}(\cdot)\right\}_{n \geq 1}$ of measurable functions s.t. $F(\omega)=\operatorname{cl}\left\{f_{n}(\omega)\right\}_{n \geq 1}$ for all $\omega \in \Omega$ (Castaing's representation)

(iii) for all $U \subseteq X$ open $\left.F^{-}(U)=\omega \in \Omega: F(\omega) \cap U \neq \phi\right\} \in \Sigma$ (in the language of measurable multifunction $\mathrm{F}^{-}(\mathrm{U})$ is called the weak inverse image of $\mathrm{V}$ under $F(\cdot))$.

A detailed treatment of measurable multifuntions can be found in Castaing-Valadier [5] and Himmelberg [6].

We denote by $S_{F}^{1}$ the set of all selectors of $F(\cdot)$ that belong to the Lebesgue-Bochner space $L_{X}^{1}(\Omega)$ i.e. $S_{F}^{1}=\left\{f(\cdot) \in L_{X}^{1}(\Omega): f(\omega) \in F(\omega) \mu-a . e.\right\}$. It is easy to see that this set is closed and it is nonempty if and oniy if $\inf _{x \in F(\omega)}\|x\| \in L_{+}^{1}(\Omega)$.

Assume that $Y, Z$ are topological spaces and $F: Y \rightarrow 2^{Z},\{\phi\}$. We say that $F(\cdot)$ is lower semicontinuous (l.s.c.) if and only if for all $V \subseteq Z$ open, $\{y \in Y: F(y) \cap$ $\mathrm{V} \neq \phi\}$ is open too.

Finally if $\left\{A_{n}\right\}_{n \geq 1}$ are nonempty subsets of $X$, we define

$$
\operatorname{sim}_{n \rightarrow \infty} A_{n}=\left\{x \in X: x=s-\lim x_{n}, x_{n} \in A_{n}, n \geq 1\right\}
$$

By $\gamma(\cdot)$ we will denote the Hausdorff measure of noncompactness i.e. if $B \subseteq X$ is bounded, then $\gamma(B)=\inf \{r>0: B$ can be covered by finitely many balis of 
radius $\leq r\}$. This is equivalent to the Kuratowski measure of nonconjactness [ 7$]$ (see also Banas-Goebel [8]). Recall that by a Kamke function we mean a function w : $[0, T] \times \mathbb{R}_{+} \rightarrow \mathbb{R}_{+}$satisfying the Caratheodory conditions (i.e. it is measurable in $t$ and continuous in $x), w(t, 0) \equiv 0$ a.e. and such that $u(t) \equiv 0$ is the only solution of the problem $u(t) \leq \int_{0}^{t} w(s, u(s)) d s, u(0)=0$.

3. EXISTENCE THEOREMS.

The setting is the following. We are given a finite interval $T=[0, b]$. On $T$ we consider the Lebesgue measure $d t$. Also let $X$ be a separable reflexive Banach space. By $X_{w}$ we will denote $X$ with the weak topology. The Cauchy problem under consideration is the following:

$$
\left\{\begin{array}{l}
\dot{x}(t) \in F(t, x(t)) \\
x(0)=x_{0}
\end{array}\right\}
$$

By a solution of $(*)$ we understand an absolutely continuous function $x: T \rightarrow X$ satisfying (*) for almost all $t \in T$.

Our first existence result is the following:

THEOREM 3.1. If $F: T x X \rightarrow P_{f}(X)$ is a multifunction s.t.

1) for all $x \in X, F(\cdot, x)$ is measurable

2) for all $t \in T, F(t, \cdot)$ is 1.s.c. from $x_{w}$ into $X$

3) for all $x \in X,|F(t, x)| \leq \psi(t)$ a.e. with $\psi(\cdot) \in L_{+}^{1}(T)$

4) for all $B \subseteq X$ nonempty and bounded we have

$$
\gamma(F(t, B)) \leq w(t \cdot \gamma(B)) \text { a.e. }
$$

where $\gamma(\cdot)$ is the Hausdorff measure of noncompactness and $w(\cdot$,$) is a$ Kanke function.

then (*) admits a solution.

PROOF: Let $r=\|\psi\|_{1}$, and consider $B_{r}\left(x_{0}\right)=\left\{x \in X:\left\|x-x_{0}\right\| \leq r\right\}$. Because of the reflexivity of $\mathrm{X}, \mathrm{B}_{\mathrm{r}}\left(\mathrm{x}_{\mathrm{O}}\right)$ is $\mathrm{w}$-compact and metrizable for the weok topology (see Dunford-Schwartz [9], theorem 3, p. 434). In the sequel we will alvays consider $B_{r}\left(x_{0}\right)$ with the weak topology. Let $L: B_{r}\left(x_{0}\right) \rightarrow P_{f}\left(L_{\lambda}^{1}(T)\right)$ be the multifunction defined by $L(x)=S_{F}^{1}(\cdot, x)$. Our claim is that $L(\cdot)$ is 1.s.c. From Delahaye-Denel [10] we know that it suffices to slow that for any $x_{n} \stackrel{W}{\rightarrow} x$ in $B_{r}\left(x_{0}\right)$ we have $s_{F(\cdot, x)}^{1} \subseteq s_{-\frac{1 i m}{n \rightarrow \infty}} S_{F\left(\cdot, x_{n}\right)}^{1}$. For that purpose let $f(\cdot) \in s_{F(\cdot, x)}^{1}$. Then $f(t) \in F(t, x)$ a.e. A straightforward application of Aumann's selection theorem can give us $f_{n}(\cdot) \in$ $S_{F\left(\cdot, x_{n}\right)}^{1}$ s.t. $\frac{d(f(t))=\left\|f(t)-f_{n}(t)\right\| \text { for all } t \in T \text {. Since } F(t, \cdot) \text { is l.s.c. }}{F\left(t, x_{n}\right)}$ 
from $x_{w}$ into $X, F(t, x) \subseteq \frac{s-\frac{\lim }{n \rightarrow \infty}}{n \rightarrow \infty}\left(t, x_{n}\right)$ and so $\lim _{n \rightarrow \infty} f(t(t))=\lim _{n \rightarrow \infty}\left\|f(t)-f_{n}(t)\right\|$ $=0$, which by the dominated convergence theorem implies that $f_{n}(\cdot) \stackrel{s-L_{X}^{1}}{\longrightarrow} f(\cdot) \Rightarrow$ $f(\cdot) \in \mathrm{s}-\frac{\mathrm{lim}}{\mathrm{n}} \mathrm{s}_{F\left(\cdot, \mathrm{x}_{n}\right)}^{1}$. So we have. shown that $s_{F(\cdot, x)}^{1} \subseteq \mathrm{s}^{-\frac{1 \mathrm{im}}{n \rightarrow \infty}} S_{F\left(\cdot, x_{n}\right)}^{1}$, which as re already said, implies the lower semicortinuity of $L(\cdot)$. Hence we can now apply theorem 3.1 of Fryszkowski [11] and deduce that there exists $\ell: B_{r}\left(x_{0}\right) \rightarrow L_{X}^{1}(T)$ continuous s.t. $\ell(x) \in L(x)$ for $x \in B_{r}\left(x_{0}\right)$. set $f(t, x)=\ell(x)(t)$ and consider the following single valued Cauchy problem

$$
\left\{\begin{array}{l}
\dot{x}(t)=f(t, x(t)) \\
x(0)=x_{0}
\end{array}\right\}
$$

Let $W=\left\{x(\cdot) \in C_{X}(T): x(t) \in B_{r}\left(x_{0}\right)\right.$ for all $\left.t \in T\right\}$ and consider the map $\Phi: W \rightarrow W$ defined by

$$
\Phi(x)(t)=x_{0}+\int_{0}^{t} f(s, x(s)) d s
$$

For $t, t^{\prime} \in T, t \leq t^{\prime}$ we have that

$$
\begin{gathered}
\left\|\Phi(x)\left(t^{\prime}\right)-\Phi(x)(t)\right\|=\left\|x_{0}+\int_{0}^{t^{\prime}} f(s, x(s)) d s-x_{0}-\int_{0}^{t} f(s, x(s)) d s\right\| \\
=\left\|\int_{t}^{t^{\prime}} f(s, x(s)) d s\right\| \leq \int_{t}^{t^{\prime}}\|f(s, x(s))\| d s \leq \int_{t}^{t^{\prime}} \psi(s) d s \\
\Rightarrow\left\|\Phi(x)\left(t^{\prime}\right)-\Phi(x)(t)\right\|<\varepsilon
\end{gathered}
$$

when $\left|t^{\prime}-t\right|<\delta$, for all $x(\cdot) \in W$. Thus ve deduce that $\Phi(W)$ is an equicontinuous subset of $C_{X}(T)$ and in fact it is uniformly equicontinuous since $T$ is a compact interval.

Also we claim that $\Phi(\cdot)$ is continuous. For that purpose let $x_{n}(\cdot) \rightarrow x(\cdot)$ in W. Then we have:

$$
\begin{gathered}
\left\|\Phi\left(x_{n}\right)(t)-\Phi(x)(t)\right\|=\left\|x_{0}+\int_{0}^{t} f\left(s, x_{n}(s)\right) d s-x_{0}-\int_{0}^{t} f(s, x(s)) d s\right\| \\
\leq \int_{0}^{b}\left\|f\left(s, x_{n}(s)\right)-f(s, x(s))\right\| d s .
\end{gathered}
$$




$$
\left\|\Phi\left(x_{n}\right)-\Phi(x)\right\|_{\infty} \rightarrow 0
$$

as

$n \rightarrow \infty$. Now consider the classical Caratheodory approximations

$$
x_{n}(t)= \begin{cases}x_{0} & \text { for } 0 \leq t \leq \frac{1}{n} \\ x_{0}+\int_{0}^{t-\frac{1}{n}} f\left(s, x_{n}(s)\right) d s & \text { for } \frac{1}{n} \leq t \leq b\end{cases}
$$

Note that for all $n \geq 1 x_{n}(\cdot) \in W$ and

$$
\left\|x_{n}(t)-\Phi\left(x_{n}\right)(t)\right\|=\left\|\Phi\left(x_{n}\right)\left(t \frac{1}{n}\right)-\Phi\left(x_{n}\right)(t)\right\| \text { for } \frac{1}{n} \leq t \leq b
$$

while $\left\|x_{n}(t)-\Phi\left(x_{n}\right)(t)\right\| \leq \int_{0}^{1 / n}\left\|f\left(s, x_{n}(s)\right)\right\| d s \leq \int_{0}^{1 / n} \psi(s) d s$ for $0 \leq t \leq 1 / n$.

Thus we have that

$$
\left\|x_{n}-\Phi\left(x_{n}\right)\right\|_{\infty} \rightarrow 0
$$

as $n \rightarrow \infty$. Let $R=\left\{x_{n}(\cdot)\right\}_{n \geq 1}$. Then since $R \subseteq(I-\Phi)(R)+\Phi(R)$ we deduce that

$R$ is uniformly equicontinuous. Set $R(t)=\left\{x_{n}(t)\right\}_{n \geq 1}$ for $t \in T$. Then we have

$$
\gamma(R(t)) \leq \gamma\left[\int_{0}^{t} f(s, R(s)) d s\right]+\gamma\left[\int_{t-\frac{1}{n}}^{t} f(s, R(s)) d s\right]
$$

Note that given $\varepsilon>0$ we can rind $n(\varepsilon)$ s.t. $\int_{t-\frac{1}{n}}^{t} \psi(s) d s<\varepsilon / 2$ for $t \in T, n \geq$

1. Hence we have that

$$
\gamma\left[\int_{t-\frac{1}{n}}^{t} f\left(s, x_{n}(s)\right) d s: n \geq n(\varepsilon)\right] \leq 2 \sup _{n \geq n(\varepsilon)}\left[\int_{t-\frac{1}{n}}^{t} \psi(s) d s\right]<\varepsilon .
$$

Using this estimate and the propoerties of $\gamma(\cdot)$ we get that

$$
\gamma[R(t)] \leq \int_{0}^{t} \gamma[f(s, R(s))] d s .
$$

Since for all $s \in T, R(s)$ is bounded, using hypothesis 4) we have that:

$$
\begin{aligned}
& \gamma(f(s, R(s))) \leq w(s, \gamma(R(s))) \text { a.e. } \\
& \Rightarrow \gamma[R(t)] \leq \int_{0}^{t} w(s, \gamma(R(s))) d s .
\end{aligned}
$$

Since $R(0)=x_{0}, \gamma(R(0))=0$ and $w(\cdot, \cdot)$ is a Kamke function we must have that 
$\gamma(R(t))=0$ for all $t \in T$. But recall (see [8]) that

$$
\gamma(R)=\sup _{t \in T} \gamma(R(t))
$$

So $\gamma(R)=0$, which means that $R$ is a relatively compact subset of $C_{X}(T)$. Therefore we can $f$ ind a subsequence $\left\{x_{n_{k}}(\cdot)=x_{k}(\cdot)\right\}_{k \geq 1}$ of $\left\{x_{n}(\cdot)\right\}_{n \geq 1}$ s.t. $\mathrm{x}_{\mathrm{k}}(\cdot) \rightarrow \mathrm{x}(\cdot) \in W$. So $\left\|\mathrm{x}_{\mathrm{k}}-\Phi\left(\mathrm{x}_{\mathrm{k}}\right)\right\|_{\infty} \rightarrow\|\mathrm{x}-\Phi(\mathrm{x})\|_{\infty}$. But we have already seen that $\left\|x_{k}-\Phi\left(x_{k}\right)\right\|_{\infty} \rightarrow 0$. Thus finally we have that

$$
\|x-\Phi(x)\|_{\infty}=0 \Rightarrow x(t)=x_{0}+\int_{0}^{t} f(s, x(s)) d s \Rightarrow x(\cdot) \text { solves }(* *) .
$$

Since the vector field of $(* *)$ is a selector of $F(\cdot, \cdot)$, we conclude that $x(\cdot)$ solves (*).

Q.E.D.

REMARK. The theorem remains true if we assume that $X$ is a separable dual space with a separable predual and $F(t, \cdot)$ is l.s.c. from $X_{w^{*}}$ into $X$.

When $X$ is finite dimensional we can have a more general boundedness hypothesis. THEOREM 3.2. If $F: T x X \rightarrow P_{f}(X)$ is a multifunction s.t.

1) for all $x \in X, F(\cdot, x)$ is measurable and $|F(t, x)| \leq a(t)\|x\|+b(t)$

a.e. with $a(\cdot), b(\cdot) \in L_{+}^{1}(T)$

2) for all $t \in T, F(t, \cdot)$ is l.s.c.

then $(*)$ admits a solution.

PROOF: Let $M=\left[\left(\left\|x_{0}\right\|^{2}+1\right) e^{2\left(\|a\|_{1}+\|b\|_{1}\right)}-1\right]^{1 / 2}$ and define the following new orientor field.

$$
\hat{F}(t, x)=\left\{\begin{array}{ll}
F(t, x) & \text { for }\|x\| \leq M \\
F\left(t, \frac{M x}{\|x\|}\right) & \text { for }\|x\|>M
\end{array} .\right.
$$

Then for every $t \in \mathrm{T}, \hat{\mathrm{F}}\left(\mathrm{t}_{,} \cdot\right)$ is the composition of the multifunction $F(t, \cdot)$ and of the M-radial retraction map $r: X \rightarrow B_{M}(0)=\{z \in X:\|z\| \leq M\}$ defined by

$$
r(x)=\left\{\begin{array}{lll}
x & \text { if } & \|x\| \leq M \\
\frac{M x}{\|x\|} & \text { if } & \|x\|>M
\end{array}\right.
$$

It is well known that $r(\cdot)$ is Lipschitz. So $\hat{F}(t, \cdot)$ is $1 . s . c$. Clearly it is measurable in $t$ and for all $x \in X,|\hat{F}(t, x)| \leq a(t) M+b(t) a . e$. So $\hat{F}(\cdot, \cdot)$ satisfies the hypotheses of theorem 3.1 (recall that $X$ is finite dimensional) and so by that theorem there exists $x: T \rightarrow X$ absolutely continuous s.t. $\dot{x}(t) \in \hat{F}(t, x(t))$ a.e., $x(0)=x_{0}$. Our goal is to show that for all $t \in T,\|x(t)\| \leq M$. We procced by contradiction. Suppose that there exist $t_{1}, t_{2} \in T s . t$. for $t \in\left(t_{1}, t_{2}\right)\|x(t)\|>M$. 
Then we have that $\dot{x}(t) \in F\left(t, \frac{M x(t)}{\|x(t)\|}\right)$ a.e. on $\left(t_{1}, t_{2}\right) \Rightarrow\|\dot{x}(t)\| \leq a(t) M+b(t)$ a.e. Set $z(t)=\|x(t)\|^{2}+1$. Then $\frac{d}{d t} z(t)=\frac{d}{d t}\left[\|x(t)\|^{2}+1\right]=2\|x(t)\| \frac{d}{d t}-\|x(t)\|$.e. Since $\frac{d}{d t}\|x(t)\| \leq\|\dot{x}(t)\|$ we get that

$$
\begin{gathered}
\frac{d}{d t} z(t) \leq 2\|x(t)\|[a(t) M+b(t)] \\
\leq 2\|x(t)\|[a(t)\|x(t)\|+b(t)] a . e \text {. on }\left(t_{1}, t_{2}\right) .
\end{gathered}
$$

Because $\|x(t)\|>M \geq 1$ on $\left(t_{1}, t_{2}\right)$ we have that

$$
\begin{aligned}
\frac{d}{d t} z(t) & \leq 2\left[a(t)\left(\|x(t)\|^{2}+1\right)+b(t)\left(\|x(t)\|^{2}+1\right)\right] \\
= & 2[a(t)+b(t)] z(t) a . e \text {. on }\left(t_{1}, t_{2}\right) .
\end{aligned}
$$

Since for $t \notin\left(t_{1}, t_{2}\right)$ we already have that $\|x(t)\| \leq M$, we can now integrate and get that for all $t \in T$

$$
z(t) \leq z(0)+2 \int_{0}^{t}(a(s)+b(s)) z(s) d s .
$$

Using Gronwall's inequality we get that for all $t \in T$

$$
\begin{gathered}
\|x(t)\| \leq\left[\left(\left\|x_{0}\right\|^{2}+1\right) \exp \left(2 \int_{0}^{b}(a(s)+b(s)) d s\right)-1\right]^{1 / 2} \\
\Rightarrow\|x(t)\| \leq M .
\end{gathered}
$$

So for all $t \in T,\|x(t)\| \leq M$. Then $\hat{F}(t, x(t))=F(t, x(t))$ which implies that $x(\cdot)$ solves the original Cauchy problem $(*)$.

$$
\text { Q.E.D. }
$$

Another existence result in this direction is the following. Again assume that $X$ is finite dimensional.

THEOREM 3.3. If $F: T x X \rightarrow P_{f}(X)$ is a multifunction s.t.

1) for all $x \in X, F(\cdot, x)$ is measurable and $|F(t, x)| \leq a(t) \psi(\|x\|) a . e$. with $\mathrm{a}(\cdot) \in \mathrm{L}_{+}^{1}$ and $\psi(\cdot)$ a positive continuous function s.t.

$\int_{t}^{\infty} \frac{1}{\psi(s)} d s=+\infty$

2) for all $t \in T, F(t, \cdot)$ is l.s.c.

then $(*)$ admits a solution.

PROOF: Because $a(\cdot) \in \mathrm{L}_{+}^{1}$ and because of our integrability hypothesis on $\psi(\cdot)$ we can find $M>\left\|x_{0}\right\| s . t . \int_{0}^{\infty} a(s) d s<\int_{\| x_{0}}^{M} \frac{1}{\psi(s)} d s$. 
Again introduce a new orientor field $\hat{F}(\cdot, \cdot)$ defined as before by

$$
\hat{F}(t, x)=\left\{\begin{array}{lll}
F(t, x) & \text { if } & \|x\| \leq M \\
F\left(t, \frac{M x}{\|x\|}\right) & \text { if } & \|x\|>M
\end{array}\right.
$$

We have already seen in the proof of theorem 3.2 that $\hat{F}(\cdot, \cdot)$ satisfies all hypotheses of theorem 3.1. So we can find $x: T \rightarrow X$ absolutely continuous s.t. $\dot{x}(t)$ $\epsilon \hat{F}(t, x(t))$ a.e. $x(0)=x_{0}$. Our claim is that $\|x(t)\| \leq M$ for all $t \in T$. Suppose not. Then we can find $t_{0} \in T$ s.t. $\left\|x\left(t_{0}\right)\right\|>M$. On the other hand we have $\|x(0)\|=$ $\left\|x_{0}\right\|<M$. Hence we can find $t^{\prime} \in T$ s.t. $\left\|x_{0}\right\| \leq\|x(t)\| \leq M$ for all $t \in\left[0, t^{\prime}\right]$. So we can write

$$
\begin{gathered}
\dot{x}(t) \in F(t, x(t)) a . e \text { on }\left[0, t^{\prime}\right] \\
\Rightarrow\|\dot{x}(t)\| \leq a(t) \psi(\|x(t)\|) a . e \text { on }\left[0, t^{\prime}\right] \\
\Rightarrow \frac{d}{d t}\|x(t)\| \leq a(t) \psi(\|x(t)\|) a . e \text { on }\left[0, t^{\prime}\right] \\
\Rightarrow \int_{\| x}^{M} \frac{1}{\overline{\psi(\|x(t)\|)} d\|x(t)\| \leq \int_{0}^{t^{\prime}} a(s) d s \leq \int_{0}^{\infty} a(s) d s} \\
\Rightarrow \int_{\left\|x_{0}\right\|^{M}}^{\frac{1}{\psi(s)}} d s \leq \int_{0}^{\infty} a(s) d s
\end{gathered}
$$

a contraciction to our choice of $M$. Thus $\|x(t)\| \leq M$ for all $t \in T$ and so $\hat{F}(t, x(t))=F(t, x(t))$, which implies that $x(\cdot)$ solves $(*)$.

Q.E.D.

REMARKS: 1) If $T=\mathbb{R}_{+}$, then we divide it into subintervals $T_{n}=[n-1, n]$. Then on $\mathrm{T}_{1}=[0.1]$ we consider the Cauchy problem $(*)$ and find a solution $x_{1}(\cdot)$. On $T_{2}=$ [1,2] ve consider again $(*)$ but with initial condition $x(1)=x_{1}(1)$. Continuing \left. this way we obtain a sequence of partial solutions ${\left\{x_{n}\right.}_{n}(\cdot)\right\}_{n \geq 1}$ defined on $T_{n} n \geq 1$. which when pieced together give us the global solution on $\mathbb{R}_{+}$.

2) If the domain of $F(\cdot, \cdot)$ is $\operatorname{TxB}_{r}\left(x_{0}\right)$, then local versions of those results are valid.

3) The above theorems as well as the one that follows extend significantly earlier results obtained by Bressan [12]. Kaczynski-Olech [13] and Lojasewic $\angle$ [14].

We will conclude this work with an existence result concerning continuous orientor-fields on a separable Banach space $X$. But first we need to introduce the concept of a semi-inner product. 
Let $X$ be a Banach space and $x^{*}$ its dual. Consider the map $J: X \rightarrow 2^{X^{*}}$ defined by $J(x)=\left\{x^{*} \in x^{*}:\left(x^{*}, x\right)=\|x\|^{2}=\left\|x^{*}\right\|^{2}\right\}$. Thanks to the Hahn-Banach theorem $J(x) \neq \phi$ for all $x \in X$. Using $J(\cdot)$ we can define the semi-inner product $(\cdot, \cdot)_{-}: \mathrm{XxX} \rightarrow \mathbb{R}$ by

$$
(x, y)_{-}=\inf \left\{\left(y^{*}, x\right): y^{*} \in J(y)\right\}
$$

For more details about semi-inner products the reader can consult Deimling [15] (p. 33).

Now let $T=[0, b]$ and let $X$ be any separable Banach space. By $h(\cdot, \cdot)$ we will denote the Hausdorff metric on $P_{f}(X)$.

THEOREM 3.4. If $\mathrm{F}: \mathrm{TxX} \rightarrow \mathrm{P}_{\mathrm{f}}(\mathrm{X})$ is a multifunction s.t.

1) for all $x \in X, F(\cdot, x)$ is measurable

2) for all $x, y \in X, h(F(t, x), F(t, y)) \leq k(t)\|x-y\|$ with $k(\cdot) \in L_{+}^{1}(T)$

3) $\mathrm{s}_{\mathrm{F}\left(\cdot, \mathrm{x}_{0}\right)}^{1} \neq \Phi$

4) for all $y \in F(t, x)(y, x)_{-} \leq c(t)\left[\|x\|^{2}+1\right]$ with $c(\cdot) \in L_{+}^{1}(T)$

then $(*)$ admits a solution.

PROOF: Let $\mathrm{M}^{2}=\left[\left\|\mathrm{x}_{0}\right\|^{2}+1\right] \mathrm{e}^{\|c\|_{1}}-1$. Again we introduce the new orientor field $\hat{F}(\cdot, \cdot)$ defined by

$$
F(t, x)=\left\{\begin{array}{lll}
F(t, x) & \text { if }\|x\| \leq M \\
F\left(t, \frac{M x}{\|x\|}\right) & \text { if }\|x\|>M
\end{array}\right.
$$

Clearly $\hat{F}(\cdot, x)$ is measurable. Also if $r(\cdot)$ is the $M$-radial retraction map, then $\hat{F}(t, x)=F(t, r(x))$. So for any $x, y \in X$ we have:

$$
\begin{gathered}
h(\hat{F}(t, x), \hat{F}(t, y))=h(F(t, r(x)), F(t, r(y))) \\
\leq k(t)\|r(x)-r(y)\| \leq 2 k(t)\|x-y\| .
\end{gathered}
$$

Furthermore note that $\left\|x_{0}\right\| S M$ and so $F\left(t, x_{0}\right)=\hat{F}\left(t, x_{0}\right)$. Hence by hypothesis

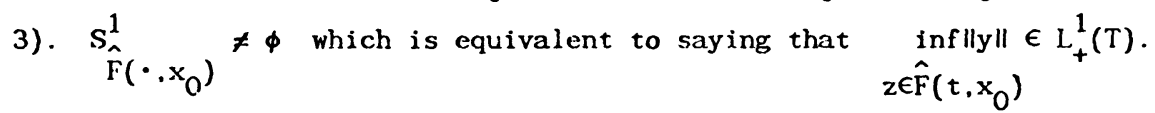

So we can apply theorem 1 of Muhsinov [16] and get that the Cauchy problem

$$
\begin{gathered}
\dot{x}(t) \in \hat{F}(t, x(t)) \\
x(0)=x_{0}
\end{gathered}
$$

admits a solution. Let $x(\cdot)$ be such a solution. We will show that $x(\cdot)$ solves the original Cauchy problem. To show that let $u(t)=\left[\|x(t)\|^{2}+1\right]$. Then we have: 


$$
\begin{gathered}
\frac{d u(t)}{d t}=2\left(\frac{d\|x(t)\|}{d t}\right)\|x(t)\| \\
\leq(\dot{x}(t), x(t))_{-} \leq c(t)\left[\|x(t)\|^{2}+1\right]=c(t) u(t) \\
\Rightarrow u(t) \leq u(0)+\int_{0}^{t} c(s) u(s) d s .
\end{gathered}
$$

Applying Gronwall's inequality we get that

$$
\begin{gathered}
u(t) \leq e^{\|c\|_{1}} u(0) \\
=\left[\left\|x_{0}\right\|^{2}+1\right] e^{\|c\|_{1}} \\
\Rightarrow\|x(t)\|^{2} \leq M^{2} \\
\Rightarrow\|x(t)\| \leq M
\end{gathered}
$$

But then from the definition of $\hat{F}(\cdot, \cdot)$ we have that for all $t \in T$

$$
\begin{aligned}
& \hat{F}(t, x(t))=F(t, x(t)) \\
& \Rightarrow x(\cdot) \text { solves }(*) .
\end{aligned}
$$

Q.E.D.

In this paper we extended the works of Kaczynski-Olech [13], Brcssan [12] and Lojasiewicz [14]. In particular theorems 3.1 and 3.4 provided infinite dimensional versions of those results, which are important in studying distributed parameter control systems, characteristic of mechanics and mathematical physics. On the other hand in theorems 3.2 and 3.3 which are finite dimensional, we have less restrictive hypotheses than [12], [13] and [14]. Specifically our orientor field $F(\cdot, \cdot)$ satisfies Caratheodory type conditions while in [12] and [14] $F(\cdot, \cdot)$ is jointly lower-semicontinuous and in [13] it is Hausdorff continuous in the state variable $x$. Furthermore our boundedness hypothesis is more general than this of [12] a:d [14] where the orientor field stays within a fixed ball of radius $M>0$, while in [13] $F(\cdot, \cdot)$ is integrably bounded.

ACKNOWLEDGMENT: I would like to thank the referee for his constructive criticism and suggestions. This work was done while the author was visiting the Mathematics Department of the University of 'Pavia-Italy. Financial support was provided by C.N.R. and N.S.F. Grant D.M.S. 8103135.

\section{REFERENCES}

1. J. P. Aubin-A. Cellina: "Differential Inclusjons" Springer, Berlin (1981).

2. F. H. Clarke: "Optimization and Nonsmooth Analys is" hiley, New York (19S3). 
3. L. Cesari: "Optimization-Theory and Applications" Springer, New York (19S.3).

4. A. Filippov: "Differential equations with discontinuous right hand side" Translations of the A.M.S. 42(1964) pp. 41-46.

5. C. Castaing-M. Valadier: "Convex Analysis and Measurable Multifunctions" Lecture Notes in Math, Vol. 580. Springer, Berlin (1977).

6. C. Himmelberg: "Measurable relations" Fund. Math $87(1975)$ pp. 52-71.

7. K. Kuratowski: Sur les espaces completes" Fund. Math 15 (1930) pp. 301-309.

8. J. Banas-K. Goebe !: "Measures of noncompactness in Banach spaces" Marcel Dekker Inc., New York (1980).

9. N. Dunford-J. Schwartz: "Linear Operators I" Wiley, New York (1958).

10. J. P. Delahaye-J. Denel: "The continuities of the point to set maps, definitions and equivalences" Math. Progr. Study 10(1979) pp. 8-12.

11. A. Fryszkowski: "Continuous selections for a class of nonconvex multivalued maps" Studia Math LXXVI(1983) pp. 163-74.

12. A. Bressan: "On differential relations with lower continuous right hand side. An existence theorem" J. Diff. Eq. 37(1980) pp. 89-97.

13. H. Kaczynski-C. Olech: "Existence of solutions of orientor fields with nonconvex right hand side" Annales Pol. Math. 29(1974) pp. 61-66.

14. S. Lojasiewicz: "The existence of solutions for lower semicontinuous orientor fields" Bul. Acad. Pol. Sc. 28(1980) pp. 483-87.

15. K. Deimling: "Ordinary Differential Equations in Banach Spaces" Lecture Notes in Math, Vol. 596. Springer Berlin (197).

16. A. Muhsinov: "On differential inclusions in Banach spaces" Sov. Math. Dokl. $15(1974)$ pp. $1122-25$. 


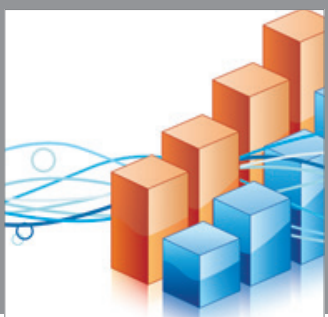

Advances in

Operations Research

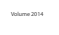

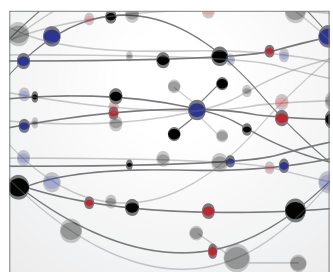

\section{The Scientific} World Journal
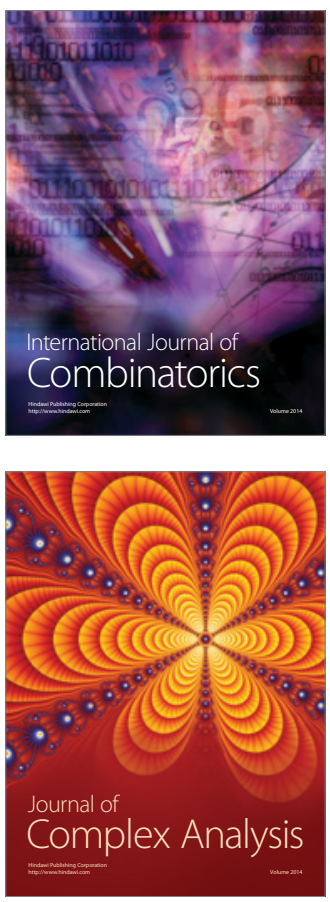

International Journal of

Mathematics and

Mathematical

Sciences
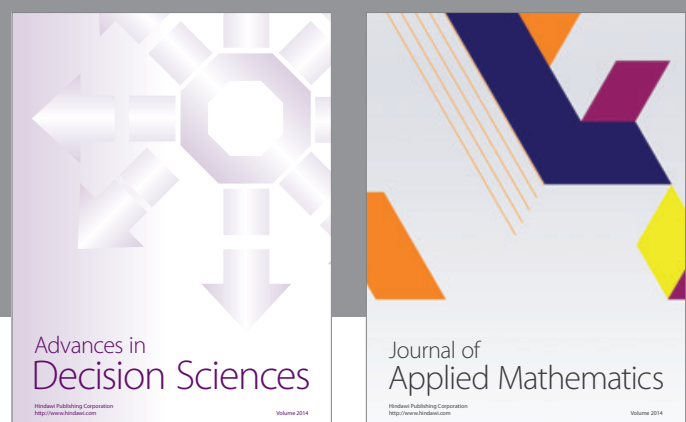

Journal of

Applied Mathematics
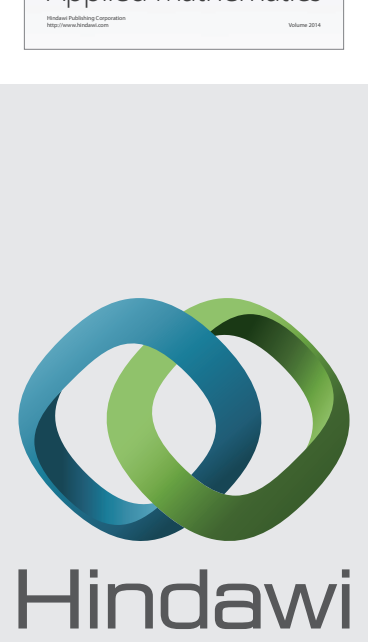

Submit your manuscripts at http://www.hindawi.com
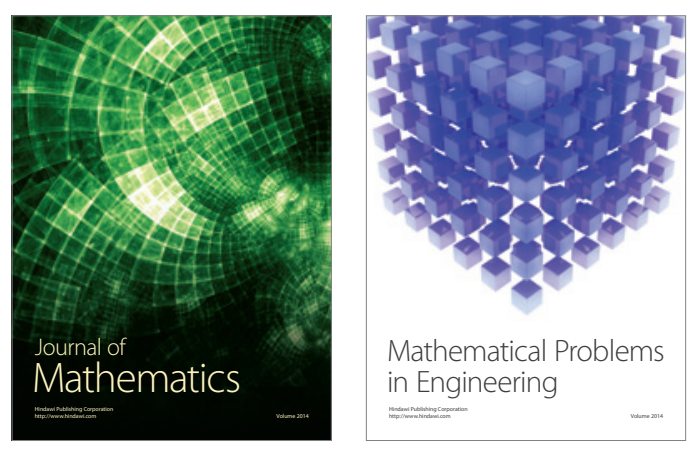

Mathematical Problems in Engineering
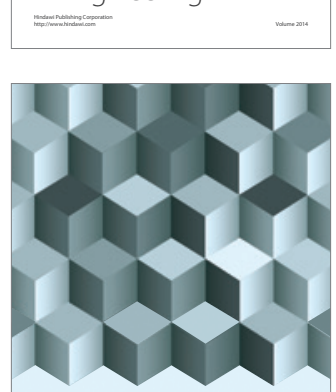

Journal of

Function Spaces
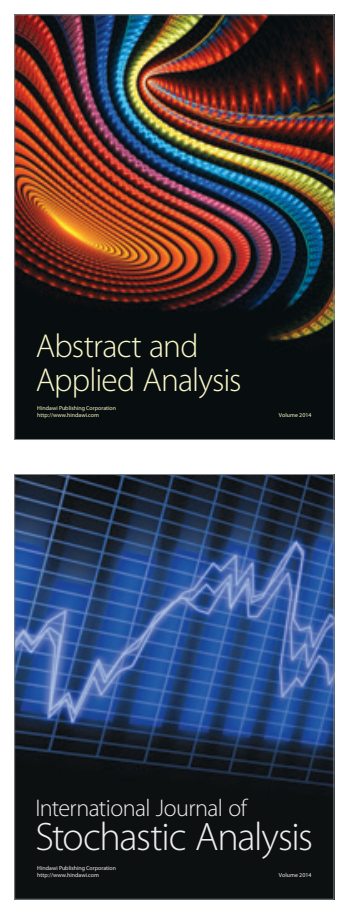

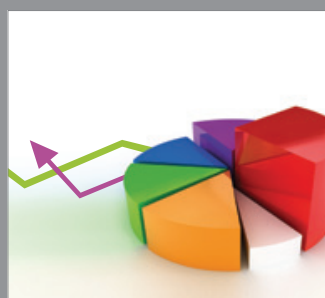

ournal of

Probability and Statistics

Promensencen
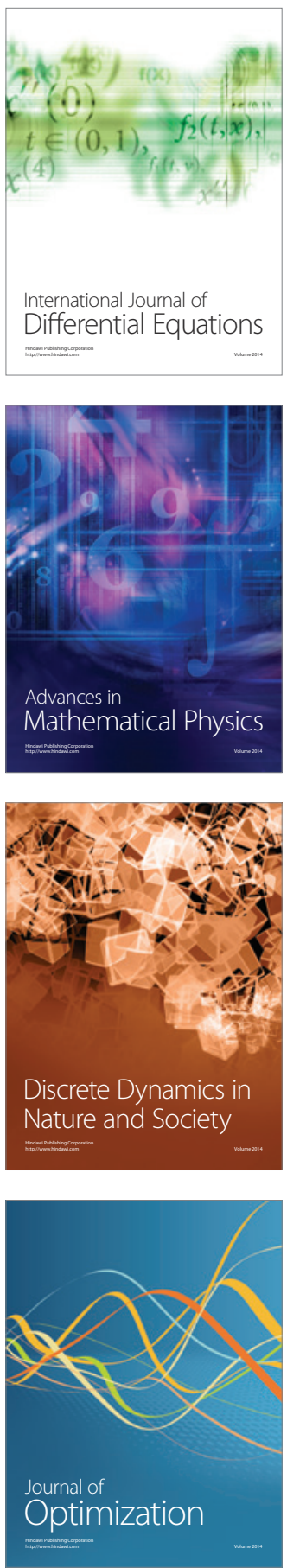